\title{
Non-IID Data Re-balancing at loT Edge with Peer-to-peer Federated Learning for Anomaly Detection
}

\author{
Han Wang \\ han.wang@ri.se \\ RISE Research Institutes of Sweden \\ Stockholm, Sweden \\ David Eklund \\ david.eklund@ri.se \\ RISE Research Institutes of Sweden \\ Stockholm, Sweden
}

\author{
Luis Muñoz-González \\ l.munoz@imperial.ac.uk \\ Imperial College London \\ London, United Kingdom \\ Shahid Raza \\ shahid.raza@ri.se \\ RISE Research Institutes of Sweden \\ Stockholm, Sweden
}

\begin{abstract}
The increase of the computational power in edge devices has enabled the penetration of distributed machine learning technologies such as federated learning, which allows to build collaborative models performing the training locally in the edge devices, improving the efficiency and the privacy for training of machine learning models, as the data remains in the edge devices. However, in some IoT networks the connectivity between devices and system components can be limited, which prevents the use of federated learning, as it requires a central node to orchestrate the training of the model. To sidestep this, peer-to-peer learning appears as a promising solution, as it does not require such an orchestrator. On the other side, the security challenges in IoT deployments have fostered the use of machine learning for attack and anomaly detection. In these problems, under supervised learning approaches, the training datasets are typically imbalanced, i.e. the number of anomalies is very small compared to the number of benign data points, which requires the use of re-balancing techniques to improve the algorithms' performance. In this paper, we propose a novel peer-to-peer algorithm,P2PK-SMOTE, to train supervised anomaly detection machine learning models in non-IID scenarios, including mechanisms to locally re-balance the training datasets via synthetic generation of data points from the minority class. To improve the performance in non-IID scenarios, we also include a mechanism for sharing a small fraction of synthetic data from the minority class across devices, aiming to reduce the risk of data de-identification. Our experimental evaluation in real datasets for IoT anomaly detection across a different set of scenarios validates the benefits of our proposed approach.
\end{abstract}

\section{CCS CONCEPTS}

- Security and privacy $\rightarrow$ Mobile and wireless security; Intrusion/anomaly detection and malware mitigation; • Computing methodologies $\rightarrow$ Machine learning.

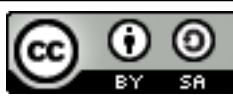

This work is licensed under a Creative Commons Attribution-ShareAlike International 4.0 License.

WiSec '21, June 28-July 2, 2021, Abu Dhabi, United Arab Emirates

(C) 2021 Copyright held by the owner/author(s).

ACM ISBN 978-1-4503-8349-3/21/06.

https://doi.org/10.1145/3448300.3467827

\section{KEYWORDS}

Federated Learning, Imbalanced Data, non-IID Data, Anomaly Detection

\section{ACM Reference Format:}

Han Wang, Luis Muñoz-González, David Eklund, and Shahid Raza. 2021. Non-IID Data Re-balancing at IoT Edge with Peer-to-peer Federated Learning for Anomaly Detection. In Conference on Security and Privacy in Wireless and Mobile Networks (WiSec '21), June 28-July 2, 2021, Abu Dhabi, United Arab Emirates. ACM, New York, NY, USA, 11 pages. https://doi.org/10.1145/ 3448300.3467827

\section{INTRODUCTION}

In recent years, because of the increased need for IoT devices, a "rush-to-market" phenomenon has occurred among the device manufacturers. Released products are usually poorly designed and there is a lack of security considerations [24], which can easily cause the IoT network to be vulnerable to attacks. Therefore, Intrusion Detection Systems (IDSs) are implemented on the IoT gateway to defend against attackers who may compromise devices within the network. An IDS learns the patterns of traffic behavior among devices and detects the anomalous patterns that deviate from normal traffic. Anomaly detection is an important research topic not only in IoT, but also in different areas and applications in system and network security, and Machine Learning (ML) techniques have become essential to address these types of problems.

ML allows to leverage the huge amount of data available from many sources and devices to extract useful knowledge and patterns and automate many tasks and processes. In anomaly detection applications, ML allows to learn and predict normal and anomalous patterns. To do this, both supervised and unsupervised learning approaches can be considered. In the first case, ML algorithms aim to learn the probability distribution for a set of normal (nonanomalous) training data points. In supervised learning settings, we have a training dataset which is typically imbalanced, i.e. the fraction of anomalous training data points is very small compared to the fraction of training data points labeled as normal.

Traditional ML approaches are centralized, which in the context of IoT implies that the data generated from IoT devices needs to be sent to the cloud or a central server for training the ML models. This can be inefficient and expensive in many cases, especially when the amount of data available is significant. In some contexts there can also be barriers to collect and process the data in a centralized way, 
due to privacy constraints and regulations, such as the ePrivacy Directive and General Data Protection Regulation (GDPR) ${ }^{1}$ in the EU and HIPAA ${ }^{2}$ in the US, and also contradict security designs, as it requires disclosure and sharing of sensitive information.

These limitations can be overcome by the use of distributed ML approaches, which allow to decentralize the training of the learning algorithms so that the training data does not need to be stored and processed in a central node. In the context of IoT, the augment of the computational power and capacity of edge devices enables the use of distributed ML approaches, pushing the computation of the models to these edge devices. Federated Learning (FL) has emerged as a very promising paradigm for training distributed ML models. FL allows to train a ML model collaboratively so that each participant in the learning task train the model locally using their own datasets for a reduced number of epochs, and then, they sent the resulting model to the central node, which merges the local models sent by the participants into a global model that is sent back to the participants. This process is repeated until some level of convergence is attained or a maximum number of training rounds is reached [13]. As the training is performed locally, the data never leaves the premises of the clients (participants) of the learning task, preserving the privacy of their datasets. The core concept of FL is "bringing the code to the data, instead of the data to the code" [4].

Despite the advantages, FL brings some new risks as well. For example, as FL relies heavily on the central node, the communication cost between the clients and the central node is one of the drawback. On the other hand, the cloud service where FL can be deployed may not be a trustworthy entity in the setup. If the cloud is compromised, every participated client gets affected. Finally, in some IoT deployments, the need of a central node to coordinate the learning process may not be possible, as the connectivity between different devices and components in the network can be limited. Recently, peer-to-peer FL (P2PFL) has caught researchers' eyes within the federated learning domain. P2PFL tries to remove the cloud and global model from the FL process, which means the clients update their local model by directly interacting with its connected neighbor clients. Roy et al. [25] first applies peer-to-peer to FL, and proposes a simple framework to fit medical application. In this work, we explore the use of peer-to-peer learning for performing anomaly detection in IoT environments. For this, we propose P2PFL, and algorithm to solve imbalanced data in anomaly detection problem in a distributed way with peer-to-peer learning.

Generally, the performance of a model relies on the training dataset which is ideally Independent and Identically Distributed (IID) over the network clients. However, in a real-world setup, the collected data varies significantly among devices since user's preferences and local environments are different. This is especially relevant for IoT anomaly detection, as the type of attacks or anomalies observed by each device can be different. This, in combination with the fact that the number of anomalies typically constitute a very small fraction of the total training set, poses a difficult challenge for the training of a distributed machine learning algorithm. It is known that even cases where the datasets are balanced. From the smaller scope, one is that the local on-device datasets are usually

\footnotetext{
${ }^{1}$ https://gdpr-info.eu/

${ }^{2}$ https://www.hhs.gov/hipaa/index.html
}

non-IID which has been shown to degrade model's performance [21]. For example, Zhao et al [33] pointed out that prediction accuracy dropped by up to $11 \%$ for MNIST and $51 \%$ for CIFAR- 10 in the case of non-IID datasets. This degradation is expected to be more severe in imbalanced dataset scenarios, which cause the model to be biased in favor of the well represented classes. From the bigger scope, the global model usually enforces a bias towards patterns provided by the majority of clients, while suppressing patterns of minor clients [27]. Duan [9] first addressed the issue of globally class-imbalanced data within the mobile domain.

In this paper, we address the problem of anomaly detection in the context IoT networks, where the data are regularly imbalanced and non-IID distributed. We leverage peer-to-peer learning, which allows to train a collaborative model without the need of a central node. We propose P2PK-SMOTE to re-balance the data by the help of P2PFL. By Taking the advantages of P2PFL, P2PK-SMOTE adaptively improves SMOTE, a state-of-the-art re-balancing technique, by generating more complex synthetic points to share some of them with other participated clients. These shared points help other clients to re-balance their own local dataset and add more variance to it, which can prevent model from degrading in non-IID settings. From the empirical results, P2PK-SMOTE performs the best in every evaluation metric comparing to other state-of-the-art data re-balancing techniques. Especially when the data is non-IID and imbalanced, P2PK-SMOTE shows its outstanding performance that reaches $100 \%$ for both precision and recall, and its false positive and false negative rate drop down to almost $0 \%$. More specifically speaking, our main contributions can be listed as follows:

- We propose P2PK-SMOTE, a fully decentralized framework that can help participated clients to re-balance local dataset for anomaly detection. P2PK-SMOTE neither require a cloud to join nor a global model to maintain by aggregating information from each client.

- We improve SMOTE adaptively to P2PFL by proposing KSMOTE that over-samples the minority class based on random $k$ points. The artificially synthetic points are generated according to fixed amount of nearest neighbors instead of only random one neighbors. This adds complexity to the synthetic points in order to prevent it from getting traced by third parties.

- We apply P2PK-SMOTE to two real-world datasets to evaluate its performance. P2PK-SMOTE proofs its effectiveness by empirical results in different settings. It especially performs well in non-IID data distribution comparing to other state-of-the-art re-balancing techniques. It can achieve a performance very close to $100 \%$ in both recall and precision, and almost $0 \%$ in both false negative and positive rate.

The remainder of the paper is organized as follows. Sec. 2 summarizes related work. Sec. 3 describes the problem, scenarios and the threat model. Sec. 4 introduces the proposed approach. Sec. 5 summarizes and discusses the experimental results across different setups. In Sec. 6, we analyze the security issues and challenges. Finally, Sec. 7 draws the conclusions and the future work. 


\section{RELATED WORK}

This section introduces some existing approaches to FL with its challenges and limitations, and briefly reviews the related work on data re-balancing.

\subsection{Federated Learning with non-IID data}

The process of training a FL algorithm includes three steps. First, an initial model is deployed from the central node (or the cloud) to each client. The model is then retrained locally by the clients using their own (private) training datasets. Once the central node requests model aggregation, for example using Federated Averaging (FedAvg) [21], selected clients send back their model update in a specific form via assigned protocols as proposed in [5]. When the global model is updated it is again deployed to the devices.

Despite the benefits brought by FL, there are still many open challenges such as (1) communication expenses or (2) systems and statistical heterogeneity [17].

The communication cost is regarded as a critical bottleneck which even takes longer time than local computation of model training. This has opened up a popular research topic regarding improved device-to-device communication. Besides Google's research team, which introduced FL to the world and has contributed to communication efficiency in FL $[14,21]$, there are many other existing works focusing on the issue with non-IID data, such as $[12,26]$. Jeong et al. [12] propose a communication-efficient algorithm for data augmentation. They utilize a knowledge distillation method whose communication payload size depends on the output dimension to reduce the overhead. Moreover, they improve FedAvg by using a Generative Adversarial Network (GAN) to reproduce local samples, and further make the training dataset to become IID.

The system heterogeneity is due to physical and environmental variability between devices. Li et al. [18] rectify FedAvg by adding a mechanism addressing the heterogeneity under non-IID data. For example, they accommodate different parameters (such as the learning rate and the number of local and global training epochs) for different devices based on their available system resources. Statistical heterogeneity can arise from non-IID data collecting in IoT networks, because of the FL setups, the number of the data points or the data distribution vary significantly across devices or clients, which would degrade the performance of the model. There are several existing works on this topic which contribute to mitigate the performance degradation due to the non-IID dataset $[9,15,29,31,33]$. Zhao et al. [33] attribute this phenomenon to the weight divergence between FedAvg and Stochastic Gradient Descent (SGD) across devices. They propose to have a globally shared dataset consisting of a uniform distribution over classes stored on the cloud. Wang et al. [29] designed a control algorithm which determines the trade-off based on the convergence bound of the gradient decent to learn the data distribution and system dynamics in real-time to minimize the learning loss.

\subsection{Re-balancing data}

Imbalanced data is a common issue in real-world classification tasks. It refers to the problem that one class is heavily under-represented compared to the other class in two-classes classification. It affects the performance of the classifier in that it leans towards the majority class, and the minor class is usually wrongly classified [6]. This scenario is typical for many cyber security applications, including anomaly, attack or fraud detection, where the datasets are often quite imbalanced. For instance, in attack detection for IoT networks, the vast majority of the traffic is benign, and only an extremely small minority of the collected traffic is malicious. General ML models have difficulties to perform well when trained with imbalanced datasets, especially for detecting samples from the minority class. To sidestep this limitation, re-balancing techniques are typically applied to improve model performance. In general, this question has been well studied for standard ML algorithms. The state-of-the-art techniques include: (1) oversampling [7, 8, 10], (2) under-sampling [10][32], and (3) penalized model [11]. Among these approaches, oversampling and under-sampling are two classic techniques to address this issue. However, the application of these techniques to FL scenarios has not been explored.

The simplest method of oversampling is random oversampling which also supplements the dataset with data points from the minority class. Chawla et al. [7] address imbalanced datasets problems by using robust oversampling. They proposed an approach that first takes the $k$ nearest neighbors for one data point from the minority class. The vector between one of these neighbors and the chosen data sample is then multiplied by a random number between 0 to 1 and added to the data sample to generate a synthetic data point. This approach is known as SMOTE. Calleja et al. [8] improved SMOTE by using weighted distance to average the neighbors in positive samples to obtain the mean as the synthetic data sample. SMOTE has shown its popularity in many use cases. However, it is not appropriate to be applied in our use case, where we propose to share synthetic examples from the minority class across different participants in collaborative learning tasks. The reason is that, typically SMOTE generates synthetic points doing an interpolation between two points. Thus, sharing synthetic data points crafted in this way, could facilitate the identification of real data points by other participants, leading to privacy breaches. We improve SMOTE to be adaptively fit in P2PFL for data re-balancing making more difficult to identify the data used by the different participants.

On the other hand, regarding to under-sampling, the simplest and most straight-forwarded way is to remove data samples from majority class randomly. However, [10] pointed out that undersampling may increase the variance and discard the important samples. [32] is one classic way to under-sampling. It under-samples the points in the majority class based on their distance to other points in the same class. Overall, these re-balancing techniques have been widely used for decades. We use them as baselines to compare to P2PK-SMOTE in our use case.

\section{PROBLEM DESCRIPTION AND SCENARIOS}

Let us consider a use case in an IoT network scenario comprising different kinds of IoT devices and an edge device, which is usually a gateway. The edge device takes the responsibility of monitoring the traffic flow and the communication between IoT network and the cloud. Poor-designed IoT device may cause security vulnerabilities that can be exploited. Mechanisms of anomaly detection are usually deployed on the edge devices to detect abnormal traffic. 
When mapping to FL setup, the edge device can be seen as the client to one center cloud. In this paper, we assume that there are $n$ clients which own data, denoted as $C=\left\{c_{1}, \cdots, c_{n}\right\}$. Each client has its on-device traffic dataset $D_{k}=\left\{x_{j}, y_{j}\right\}_{j=1}^{\left|D_{k}\right|}$ where $\left|D_{k}\right|$ is the total number of data samples in the dataset on client $k, x_{j}$ indicates the $j$-th sample, and $y_{j}$ is the corresponding label. Specifically, $y_{j} \in Y=\{1, \cdots, L\}$, where $L$ represents the number of labels. The general training process of FL can be formulated as minimizing the global loss function $[17,19]$ :

$$
\begin{array}{r}
\min _{w} \sum_{k=1}^{n} p^{k} F^{k}(w), \\
F^{k}(w)=\frac{1}{\left|D_{k}\right|} \sum_{j_{k}=1}^{\left|D_{k}\right|} f_{j}^{k}\left(w ; x_{j}^{k}, y_{j}^{k}\right)
\end{array}
$$

where $p^{k}$ denotes the related impact of client $k, f^{k}$ is the local objective function for the $k$ th client to detect the anomalies. As we focus on studying class-wised imbalance in non-IID datasets, we consider two subsets for every client: $D_{\text {min }}^{k} \subset D_{k}$ and $D_{\text {maj }}^{k} \subset D_{k}$, where $D_{\text {min }}^{k}$ is the set of data samples from minority class on client $k$, and $D_{m a j}^{k}$ is the set for the majority class on client $k$. Depending on the scenario, there can be various setups for $D_{\min }$ and $D_{m a j}$.

\subsection{Scenarios}

In this paper we analyze anomaly detection in IoT networks, considering only binary classification problems that include benign and malicious traffic. In this context, there are four scenarios as listed below.

Scenario 1 - Globally balanced and IID dataset: this is the simplest situation where the local datasets for all the participants are IID-distributed and balanced, i.e. $\left|D_{m a j}\right|$ is similar to $\left|D_{\text {min }}\right|$. From the global perspective, the data is balanced as well.

Scenario 2 - Globally imbalanced and IID dataset: For every client, the benign traffic is $D_{m a j}$ and malicious traffic is $D_{\text {min }}$. All traffic are IID-distributed across clients. The global dataset considering the data from all participants is imbalanced, i.e. $\left|D_{\min }\right| \ll$ $\left|D_{m a j}\right|$.

Scenario 3 - Globally imbalanced and mixed IID dataset: the datasets from the clients are from the same distribution, but the imbalance ration across the clients is different, i.e. the proportion of benign and malicious examples is not the same. For instance, there can be some clients with more malicious traffic than benign, which means $D_{m a j}$ is malicious traffic, and $D_{\text {min }}$ is benign traffic. On the other hand, for some other clients we can have the opposite. This scenario covers cases where some devices are compromised most of the time during the data collection phase.

Scenario 4 - Globally imbalanced and non-IID dataset: We have similar setup as in Scenario 2, but similar to many real-world settings, we consider the case where the distribution of benign and malicious traffic varies across devices, and thus, across clients. Thus, each client has its particular data distribution.

\subsection{Threat Model}

The adversary to our method are the participating compromised IoT devices. We aim to detect the malicious traffic generated from these devices in order to isolate them as early as possible. Moreover, compared to traditional FL, there is no central node/cloud needed to coordinate the participated clients, and no need to maintain the global model that aggregates information from participants. Thus, we have less entities to trust, and less information to share. In our peer-to-peer FL setup, we only need to ensure the trust between participated clients. In addition, we assume that vendors or manufactures of IoT devices are not malicious, which means the device is benign when first joining the IoT network.

\section{APPROACH}

In this section, we describe P2PK-SMOTE, our proposed method for training ML-based anomaly detection systems in IoT environments with peer-to-peer (P2P) learning.

\subsection{System Design}

Fig. 1 depicts how our system works. Within an IoT network, P2PKSMOTE can be employed on the edge devices, which is seen as the client in FL set up. The edge device can be a gateway that monitors the network traffic in order to detect any abnormal behavior. In contrast to traditional FL, in P2PK-SMOTE, the cloud, which is the central node, does not participate or orchestrate the training process except for the deployment of the initial model. Overall, P2PK-SMOTE consists of three phases: 1) re-balancing, 2) optimization of the anomaly detector, and 3) sharing. In the first phase we re-balance the training data in the clients (devices) with over-sampling (i.e. augmenting the number of data points from the minority class), by generating synthetic data points from the genuine data points in the minority class. The synthetic data points are fed into the anomaly detector together with the original dataset owned by the client so that the fraction of benign and malicious data points is similar. Then, the anomaly detector is optimized with this combination of genuine and synthetic data points. Finally, the resulting model and a fraction of the synthetic data points generated are shared with a subset of the connected clients. It is important to note that the clients are not sharing directly training data points, but synthetic data generated from the distribution of training points available from the minority class. Although this can reveal some properties about the data of the clients, in scenarios where the clients can be trusted (e.g. they belong to the same organization) the benefits of sharing this information for building a more accurate model, especially in the case of non-IID scenarios, outweighs the potential risks for leaking of information. In the following sections, we explain the process in more detail.

\subsection{K-SMOTE Re-balancing}

Regarding data re-balancing techniques, Synthetic Minority OverSampling Technique (SMOTE) [7] has been seen as the state-of-theart method. It is widely used in many imbalanced data problems. SMOTE generates artificial data by linear interpolation of samples in the minority class $D_{\min }$. Concretely, SMOTE first utilizes KNearest Neighbors $(\mathrm{KNN})$ for every $x_{i} \in D_{\min }$. It then randomly selects one neighbor to apply linear interpolation with a random value between 0 and 1 . The new synthetic data is given as follows:

$$
x_{\text {syn }}=x_{i}+\left(\hat{x}_{i}-x_{i}\right) \times \delta
$$




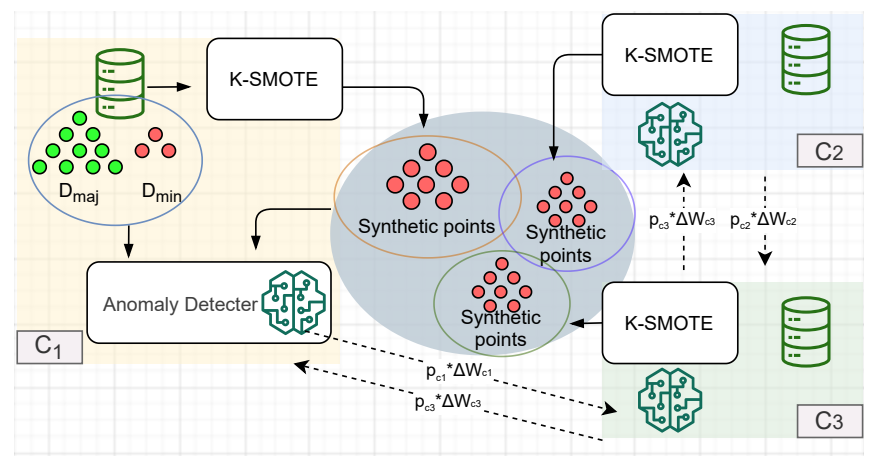

Figure 1: System overview of P2PK-SMOTE: Each client contains its local dataset, K-SMOTE, and a local model for anomaly detection. The local dataset contains samples from both the majority (green dots) and the minority (red dots) classes.

where $\hat{x}_{i}$ and $x_{i}$ are both from $D_{\min }, \hat{x}_{i}$ is a random sample from the set of $k$ nearest neighbors of $x_{i}$ and $\delta$ denotes a random number in $[0,1]$. The synthetic data point $x_{s y n}$ will be added to the dataset for training.

Although SMOTE works well in many use cases, it is not efficient for re-sampling based on every data point in the minority class. We propose K-SMOTE to reduce its computational complexity by only randomly selecting $K$ number of samples in $D_{\min }$. Moreover, instead of randomly choosing one of the $k$ nearest neighbors, we take all $k$ nearest neighbors, denoted as $N=\left\{\hat{x}_{1}, \cdots, \hat{x}_{k}\right\}$, to apply linear interpolation to generate the resulting synthetic data point. This way, when sharing synthetic data points across different clients, the identification of genuine data points from the synthetic examples is more difficult than in the case of linear interpolation between two data points. The formulation can be re-written as:

$$
x_{s y n}=x_{i}+\frac{1}{|N|} \sum_{n=1}^{|N|}+\left(\hat{x}_{n}-x_{i}\right) \times \delta_{n}
$$

where $\hat{x}_{n}$ is one of the nearest neighbors from KNN.

Algorithm 1 describes the whole process of K-SMOTE in detail. First, the input to the algorithm consists of the dataset $D$, that includes $D_{m a j}$ and $D_{m i n}$, and several hyper-parameters including: the number of base data to re-sample $k$, and the ratio $r$ to define the number of synthetic points to be created. The output is the set of synthetic points. K-SMOTE first randomly picks $k$ number of base data (line 2). For every base data $x_{b}$, K-SMOTE creates $n s k$ synthetic points based on set of nearest neighbors $N$. In line 9 shows the linear interpolation that is as the same as Equation 3. The process of generating synthetic points is described from line 5 to line 13 in Algorithm 1.

\subsection{Peer-to-Peer Data Re-balancing in FL}

Peer-to-Peer (P2P) learning brings some benefits from security perspective. Without the coordination of central node, it not only reduces the communication overhead, but also reduces the exposure of model to every other clients. In P2P setting, the local model on a client is only shared with other adjacent clients.

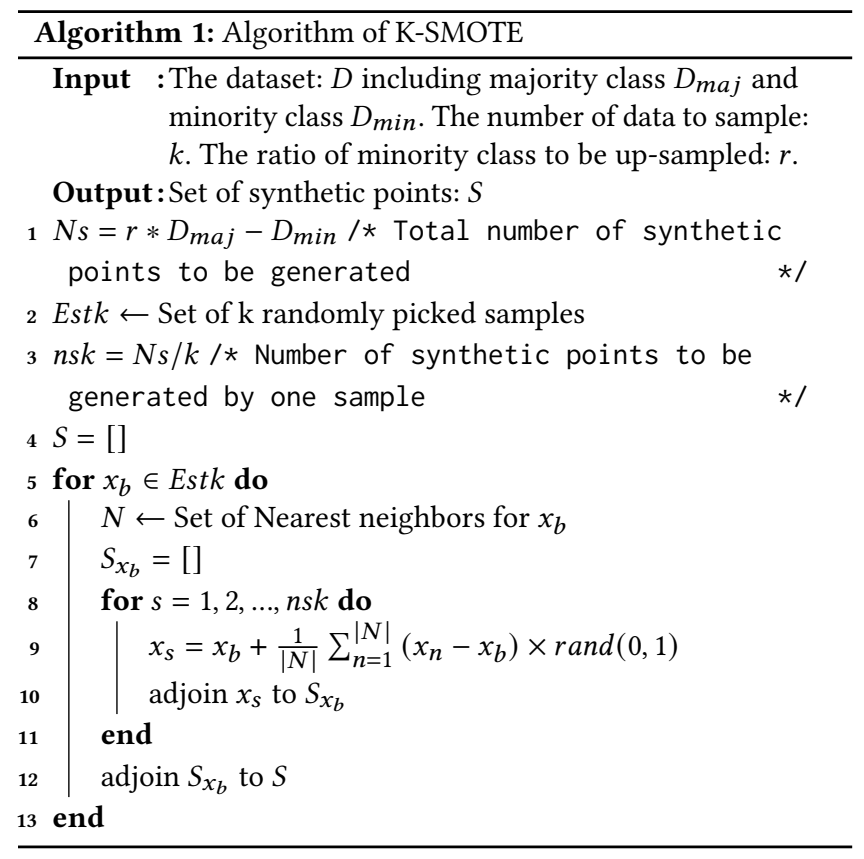

P2PK-SMOTE provides a framework for every client to re-balance its own local dataset by the help from the synthetic points provided by other clients. Moreover, P2PK-SMOTE tries to minimize every loss function on different clients with their local datasets after Fe$\mathrm{dAvg}$. Therefore, the weights of local models on each client $c_{k} \in C$ are optimized by gradient descent with learning rate $\eta$. Expanded from Equation 1, the weights of the local models are updated regularly every $t$ epochs during training. This can be formulated as:

$$
w_{t+1}^{k}=w_{t}^{k}-\eta \nabla_{w_{t}^{k}} \frac{1}{\left|D_{k}\right|} \sum_{i=1}^{\left|D_{k}\right|} L\left(w_{t}^{k} ; x_{i}^{k}, y_{i}^{k}\right)
$$

where $w_{t+1}^{(k)}$ indicates the weight on client $k$ in $(t+1)$-th epoch, and $L$ is the loss function. When a random client calls for model merging, which includes two phases: FedAvg and sharing synthetic points. When applying FedAvg, P2PK-SMOTE chooses one random client $g$ to update. The update function can be formulated as:

$$
\begin{aligned}
w_{t+1}^{g} & =\sum_{k=1}^{\left|C_{\text {sub }}\right|} p^{k} w_{t+1}^{k} \\
& =\sum_{k=1}^{\left|C_{\text {sub }}\right|} p^{k} w_{t}^{k}-\frac{\eta}{\left|D^{k}+D_{\text {syn }}^{k}\right|} \nabla_{w_{t}^{k}} \sum_{i=1}^{\left|D^{k}+D_{\text {syn }}^{k}\right|} L\left(w_{t}^{k} ; x_{i}^{k}, y_{i}^{k}\right)
\end{aligned}
$$

where $\left|C_{s u b}\right|$ represents the subset of clients which is connected to client $g$, and $\left|D^{k}+D_{\text {syn }}^{k}\right|$ indicates the number of the union set including its local data and synthetic points. Specifically, $p^{k}$ denotes the weight of client $k$, it considers the data variance and data size on the local dataset. This is defined as:

$$
p^{k}=\lambda \times \operatorname{Variance}\left(D_{\text {base }}^{k}\right)+(1-\lambda) \times \text { DataSize }\left(\left(D_{\text {base }}^{k}\right)\right.
$$


where Variance () indicates the function to measure the data variance of $D_{\text {base }}^{k}$; DataSize () represents the function to measure the size of the local dataset. $\lambda$ denotes the trade-off between the data variance and the data size.

After averaging, client $g$ will collect some of random synthetic points generated by every client $k \in C_{s u b}$. This can be expressed as:

$$
D^{g} \cup \bigcup_{k=1}^{\left|C_{\text {sub }}\right|} D_{\text {syn }}^{k} \times \alpha
$$

where $\alpha$ represents a ratio of the number of synthetic points to be randomly chosen from $D_{\text {syn }}^{k}$.

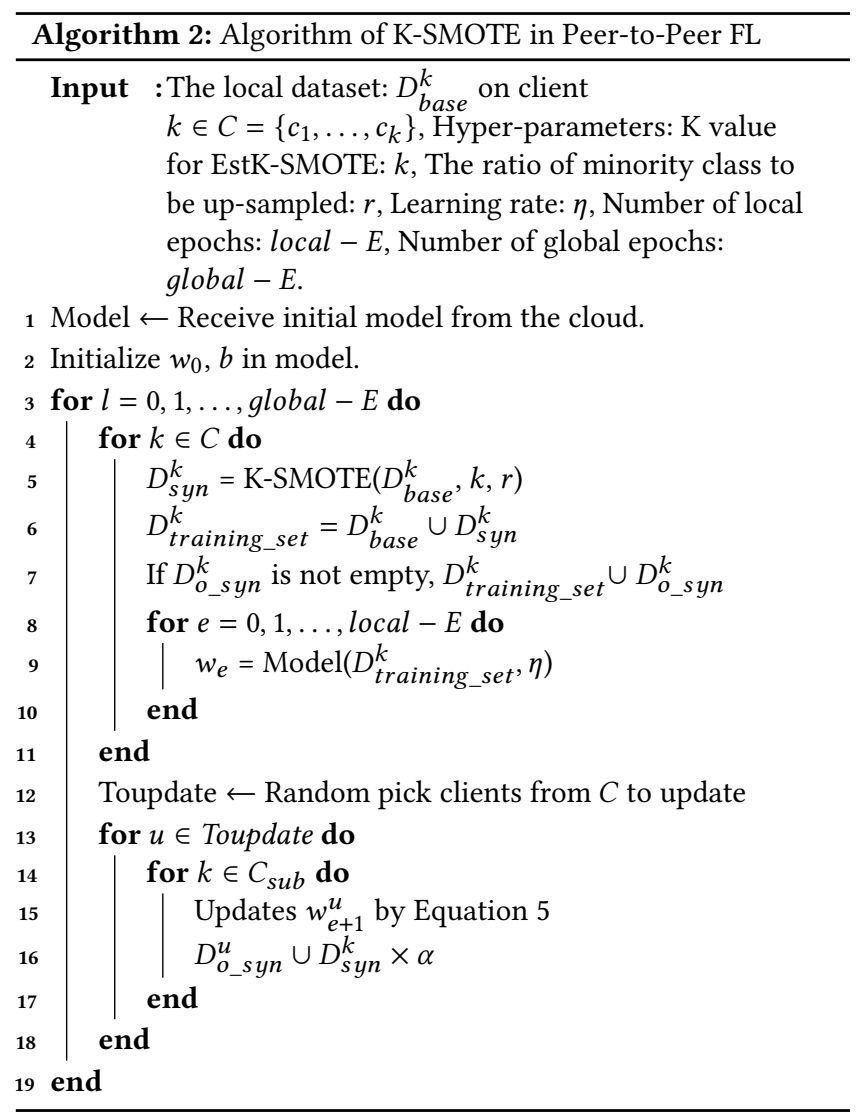

Algorithm 2 shows the P2P learning process of P2PK-SMOTE. The input data to P2PK-SMOTE includes: the local dataset $D_{b a s e}^{k}$ on client $k$, some hyper-parameters such as $k$ and $r$, which are the input to K-SMOTE, learning rate $\eta$, number of global and local epochs. In the very beginning, an initial model will be received from the cloud in line 1 . Here is only the time when cloud joining the process. Therefore, we assume the model is benign. Next, every client trains local model within a specific local epochs. Before starting the training process, the K-SMOTE is applied to generate synthetic points $D_{\text {syn }}^{k}$ for the client $k$, which is described in line 5 . Then $D_{\text {syn }}^{k}$ along with the local dataset are fed to the model for training (line 9). After every client finishes the local training, P2PK-SMOTE randomly picks random number of clients to update their local model. As

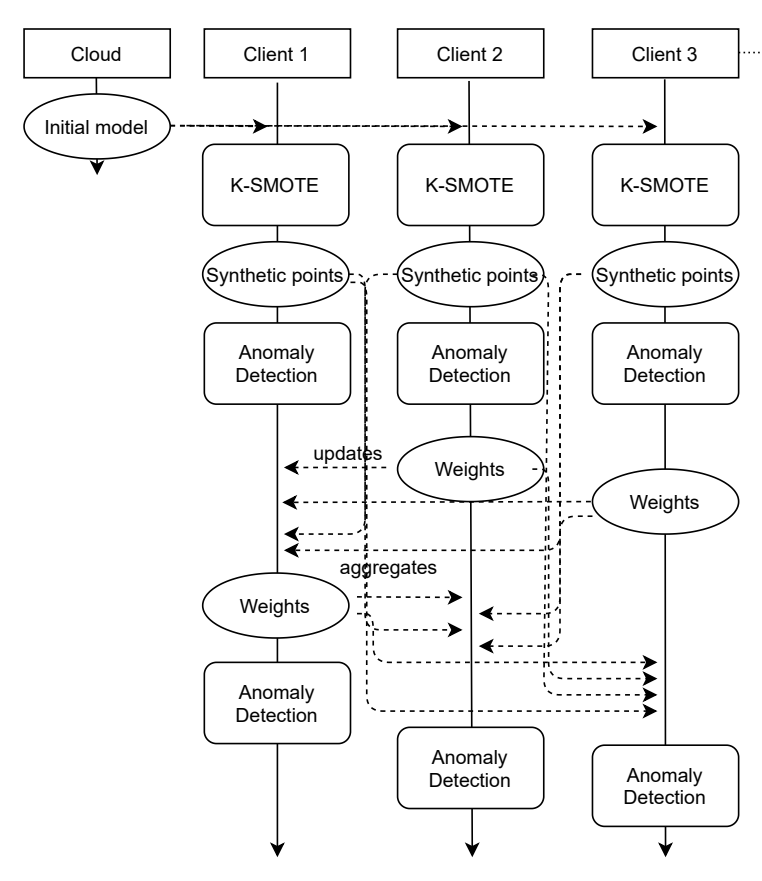

Figure 2: The workflow for P2PK-SMOTE

shown in line 13 to 16 in Algorithm 2, every picked client updates there weight by Equation 5, and receives other synthetic points $D_{o_{-} \text {syn }}^{k}$ provided by other $k$ clients. The P2P training will be end after the given global epochs. Fig. 2 graphically summarizes the workflow process of P2PK-SMOTE.

\section{IMPLEMENTATION AND EVALUATION}

We implement P2PK-SMOTE in Python. We utilize Pytorch that is a ML library especially for deep learning. With the help of Pytorch, we implement K-SMOTE and feed-forward neural network from scratch. We choose to use Scikit-leran tools for data pre-processing such as splitting the dataset, and Imblearn tools to implement regular SMOTE as our baseline approach. Regarding applying P2P FL, we implement our aggregation algorithm from scratch.

\subsection{Experiment Setup}

We conduct experiments on a Kubernetes cluster equipped with Nvidia-Gtx-2080ti GPU and 10 GB CUDA memory on the cloud service provided by RISE ICE.

5.1.1 Dataset: N-BaIoT dataset consists of real-world network traffic flows from 9 commercial IoT devices. More details of these 9 devices can be found in [22], including 4 security cameras (Provision PT 838 Security Camera, Provision PT737E Security Camera, SimpleHome XCS7 1002 WHT Security Camera, and SimpleHome XCS7 1003 WHT Security Camera), 2 doorbells (Danmini, and Ennio), a Ecobee thermostat, a Philips B120N/10 baby monitor, and a Samsung SNH $1011 \mathrm{~N}$ webcam. There are 23 incremental statistics features extracted from 5 different time windows $(100 \mathrm{~ms}$, $500 \mathrm{~ms}, 1.5 \mathrm{sec}, 10 \mathrm{sec}$, and $1 \mathrm{~min}$ ). This dataset is collected for detecting Botnet in IoT network. It captures both benign traffic and 
malicious traffic carried by 2 botnets (Mirai, BASHLITE). We choose the malicious traffic from Mirai as our abnormal traffic.

UNSW BoT-IoT dataset is created by designing a realistic network environment in the Cyber Range Lab of The center of UNSW Canberra Cyber. Koroniotis et al. [16] set up a testbed environment to simulate a real-world IoT network that is under Botnet attack. They launch Virtual Machines (VMs) to simulate the network behavior of IoT devices. The devices include a weather station, a smart fridge, a motion activated lights, a remotely activated garage door, and a smart thermostat. They record both normal/benign and botnet/anomaly traffic from all these devices. It is captured in PCAP files that are $69.3 \mathrm{~GB}$ in size, with more than $72,000,000$ records. The attack traffic include Distributed Denial-of-Service attack (DDoS), DoS, OS and Service Scan, Key-logging and Data exfiltration attacks. They extract statistical features from PCAP, and come up 10 best features. More details about this dataset can be found in [16]. In this paper, we use the top 10 features for the following experiments.

5.1.2 Evaluation metrics: The general evaluation metrics for classification such as accuracy are ambiguous and misleading in the case of problems involving imbalanced datasets, as the accuracy shows the ratio of correct classified among the whole dataset, which it easily biases towards to the majority class. In order to have an overall evaluation, we therefore choose different four metrics to measure model's performance. we first investigate the False Negative Rate (FNR) of the local model on every client. FNR shows the ratio of the model making misclassification on the positive points. By analogy of anomaly detection, low FNR means the model can detect anomaly very well. It is formulated as: $F N R=\frac{F N}{F N+T P}$, where $F N$ denotes the number of false negatives, and $T P$ denotes the number of true positives. On the other hand, we take False Positive Rate (FPR) into account. FPR shows the ratio of the model making misclassification on the negative points. It is formulated as: $F P R=\frac{F P}{T N+F P}$, where $F P$ denotes the number of false positives, and $T N$ denotes the number of true negatives. Moreover, we check the recall value when the precision reaches $75 \%$, and precision value when the recall reaches $75 \%$ for each local model. Regarding to the scenarios of global balanced dataset, we use the Area Under Curve (AUC) and the F1-score to evaluate the model.

5.1.3 Model: In the following sets of experiments, we evaluate $\mathrm{P} 2 \mathrm{PK}-\mathrm{SMOTE}$ in the $\mathrm{P} 2 \mathrm{P}$ situation where all the clients are completely connected. We first configure the model to be a feed-forward neural network with 4 hidden layers with dropout and batch normalization. We choose Leaky ReLU to be the activation function for the first 3 layers, and Sigmoid for the last one. We set Adam as the optimizer to the model with learning rate that is $\eta=1 e-5$ and weight decay $1 e-3$. The hyper-parameter such as the input $k$ to K-SMOTE is set to 15 , and for each $k$ samples, we consider its $N=20$ nearest neighbors to over-sample the minority class $D_{\text {min }}$. And, we set $\lambda$ to 0.8 which is the trade-off controlling the weight of the clients. The weight of one client $p^{k}$ considers the relative data size and data variance with its connected clients. So the weights from all connected clients $p^{k}$ sum to 1 . In addition, we set the number of training local epochs at each client to 5 , and there are various setup for different experiment such as communication epochs and the probability of an element to be zeroed $p$ for dropout.

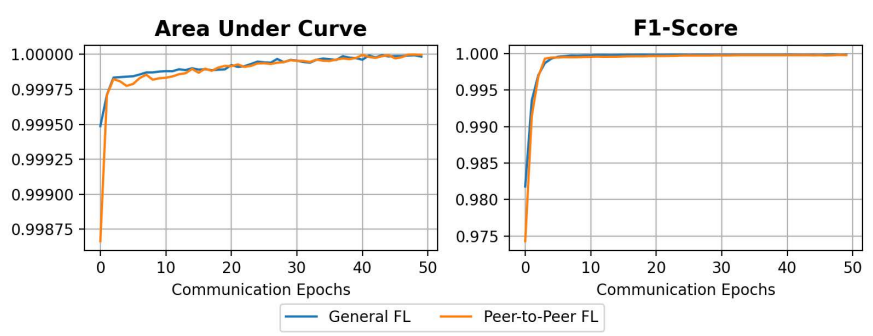

Figure 3: General FL v.s. Peer-to-Peer FL in Experiment 5.2.1

Table 1: Traffic distribution on clients for Experiment 5.2.2

\begin{tabular}{|c|c|c|c|}
\hline \multicolumn{4}{|c|}{ N-BaIoT - PT 838 Security Camera } \\
\hline & Client A & Client B & Client C \\
\hline Training_benign & 11821 & 11821 & 15762 \\
\hline Training_anomaly & 23 & 34 & 58 \\
\hline Local_Val_benign & 17732 & 17732 & 23643 \\
\hline Local_Val_anomaly & 208 & 312 & 521 \\
\hline \multicolumn{4}{|c|}{ UNSW BoT-IoT } \\
\hline & Client A & Client B & Client C \\
\hline Training_benign & 79 & 90 & 68 \\
\hline Training_anomaly & 6815 & 10905 & 4089 \\
\hline Local_Val_benign & 35 & 59 & 47 \\
\hline Local_Val_anomaly & 4847 & 4154 & 5539 \\
\hline
\end{tabular}

\subsection{Results and Discussion}

Our evaluation consists of four set of experiments regarding to four scenarios: (1) Globally balanced and IID data, (2) Globally imbalanced and IID data, (3) Globally imbalanced and mixed IID dataset, and (4) Globally imbalanced and non-IID dataset.

5.2.1 Globally balanced and IID data: We first investigate how P2PFL performs against standard FL. In this experiment, We choose $\mathrm{N}$-BaIoT to evenly distribute the similar amount of data in both classes to 3 clients. Therefore, there is no majority nor minority class. We simply measure a binary classification problem on both kind of FL.

As shown in Fig. 3, we easily find that there is no huge difference of model performance between general FL and P2PFL, except for that P2PFL converges a little slower than general FL. This phenomenon is expected because P2PFL makes clients easier to be affected by each other. Despite of this, P2PFL does not need a central node or cloud to be a coordinator who is maintaining a global model collaboratively trained by all the clients.

5.2.2 Globally imbalanced and IID data: In this set of experiments, we first measure the influence brought by imbalanced dataset to the model by using N-BaIoT dataset. We distribute the traffic of PT 838 Security Camera from N-BaIoT to 3 clients with different fraction of benign and malicious data. The data distribution is shown in Table 1 . As Table 1 shows, both training set and local validation set are extremely imbalanced and skewed to benign traffic. All the results from the following experiments are obtained from the local validation set on each client. 
False Negative Rate

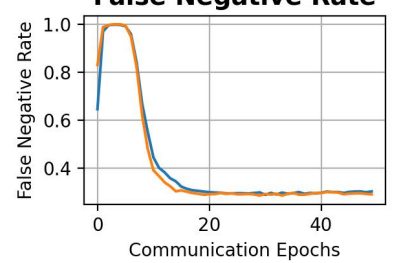

Precision_@Recall75

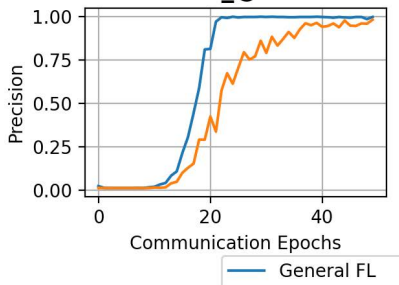

- General FL
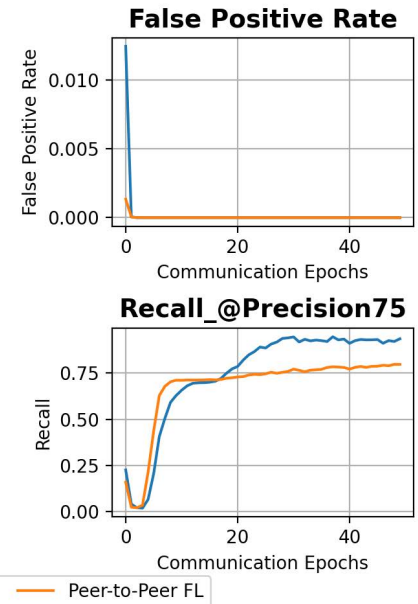

Figure 4: Comparison of different metrics in Experiment 5.2.2 between general FL and peer-to-peer FL

We use FNR, FPR, precision and recall to evaluate the model performance on imbalanced dataset. And the result is shown as the average from every client. As Fig. 4 suggests, if we do not re-balance the dataset, the model is barely able to detect the anomaly traffic. FNR and Recall in Fig. 4 is converged at around $0.4 \%$ and $0.75 \%$ respectively, which means the prediction of the model leans toward benign traffic that is the majority class in the dataset. Thus, the data re-balancing techniques are required to make model be trained better. From Fig. 4, P2PFL performs almost the same as general FL. It suggests that we apply P2PFL to the following experiments.

To evaluate P2PK-SMOTE, we compare it to other 3 most widely used re-balancing techniques as baselines. They are random oversampling, random down-sampling, and SMOTE. We take both $\mathrm{N}$ BaIoT and UNSW Bot-IoT datasets into account for evaluation. The data distribution for UNSW Bot-IoT is listed in Table 1. In contrast to N-BaIoT dataset, UNSW Bot-IoT dataset has more attack/anomaly traffic. Thus, both training set and local validation set are extremely imbalanced and skewed to anomaly traffic.

Fig. 5 shows the overall comparison of performance among the baselines and P2PK-SMOTE by useing N-BaIoT dataset. As shown in the figure, P2PK-SMOTE performs the best in every evaluation metrics. FNR and FPR of P2PK-SMOTE are dropped down to $0 \%$, and precision and recall reach $100 \%$. It is worth mentioning that SMOTE and over-sampling seems to be over-fitting in the training set, as shown their performance in FNR and recall. Both of these two baselines raise in FNR, and drop in recall in the end of training.

Fig. 6 shows the results based on the UNSW BoT-IoT dataset. Since this dataset has more attack/anomaly traffic than normal/benign traffic, the classifier is bias toward to anomaly traffic which is reflected on FPR value and recall. As shown in Fig. 6, when the data is not re-balanced, the FPR is stuck at very high value. Nevertheless, comparing P2PK-SMOTE to other baselines, P2PK-SMOTE shows better results in FNR and precision, where FNR stays at $0 \%$ and precision reaches $100 \%$.
Table 2: Traffic distribution on clients for Experiment 5.2.3

\begin{tabular}{|c|c|c|c|}
\hline \hline & Client A & Client B & Client C \\
\hline Training_benign & 54 & 75 & 9721 \\
Training_anomaly & 2131 & 2486 & 21 \\
\hline Local_Val_benign & 151 & 64 & 19486 \\
Local_Val_anomaly & 2150 & 2458 & 30 \\
\hline
\end{tabular}

5.2.3 Globally imbalanced and mixed IID data: In this experiment, we investigate how P2PK-SMOTE performs in the situation when there are different setups of majority and minority class. Practical speaking that some clients, that is IoT network, are under flooding attack, which means the anomaly traffic is the majority; some other clients may have some passive attacks that are harder to detect. Therefore, in this experiment, we distribute the data of PT 838 Security Camera from $\mathrm{N}-\mathrm{BaI}$ T dataset to 3 clients according to Table 2. As shown in the table, Client A and B have more anomaly traffic than benign traffic; Client $C$ has more benign than anomaly.

Fig. 7 shows the comparison among baselines and P2PK-SMOTE. It shows that every method works well in this experiment even we do not re-balance the data. We speculate the reason is that the dataset is globally balanced, so the classifiers have better results in every method. However, when we look closer to the FNR value and precision of P2PK-SMOTE, we find that it stays at $0 \%$ in FNR, and has more stable and slightly better performance in precision.

5.2.4 Globally imbalanced and non-IID data: In this experiment, we investigate the most challenging problem in this paper, which is our main focus. We study how P2PK-SMOTE can solve the case that is the closest to the real-world set up. In IoT network, the data are heterogeneous among different devices, so the data are in non-IID distribution. When especially in the problem of anomaly detection in IoT network, the data are usually non-IID and imbalanced. So, we first choose 3 different devices (PT 838 Security Camera, XCS7 1002 WHT Security Camera and Danmini Doorbell) from $\mathrm{N}-\mathrm{BaIoT}$ dataset to distribute to 3 clients. Each client has totally different traffic both in benign and anomaly. We then randomly pick some data from each device which are not in training set to create a global test set that is to evaluate each local model. Regarding to UNSW BoT-IoT dataset, we pick 3 different attack traffic (Service Scan, DoS and DDoS), and mix up normal traffic to distribute to 3 clients. It simulates that every client is under different kind of attack. Contrary to N-BaIoT, UNSW BoT-IoT is skewed to anomaly traffic. The distribution is listed in detailed in Table 3.

The empirical results are shown in Fig. 8 and Fig. 9. Obviously finding the problem is so complicated that every method is hard to fast converge, so we set our models to train 300 communication epochs on every client. As shown in Fig. 8, P2PK-SMOTE performs apparently the best in precision and recall on N-BaIoT dataset. Along the training process, FNR and FPR are also converged to near $0 \%$. Note that although over-sampling has consistent low in FNR value, its FPR is converged at around 50\%. Among the other techniques, P2PK-SMOTE show its fast convergence in FNR and FPR. On the other hand, P2PK-SMOTE also proofs its effectiveness on UNSW BoT-IoT dataset shown in Fig. 9. Compare to other methods, FNR of P2PK-SMOTE keeps $0 \%$, and its FPR converges to near $0 \%$ 

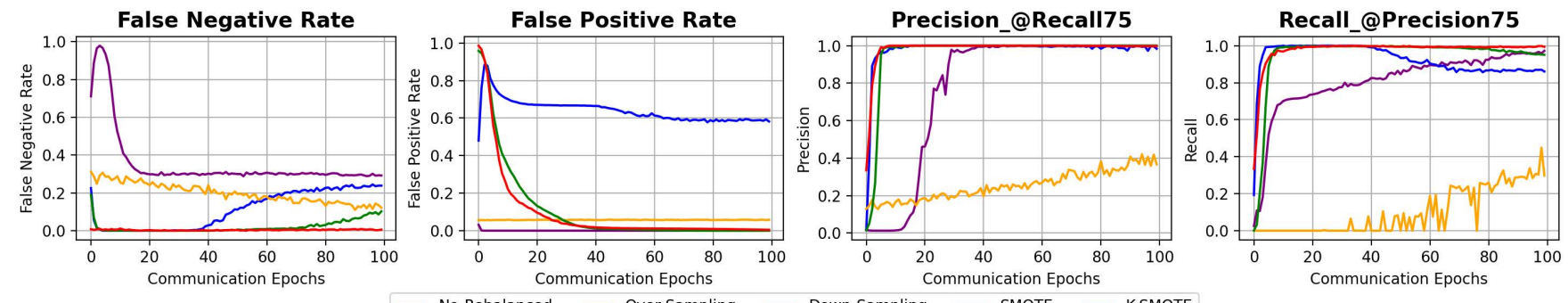

- No-Rebalanced - Over-Sampling — Down-Sampling — SMOTE - K-SMOTE

Figure 5: P2PK-SMOTE versus other state-of-the-art methods on different metrics in Experiment 5.2.2 on N-BaIoT dataset
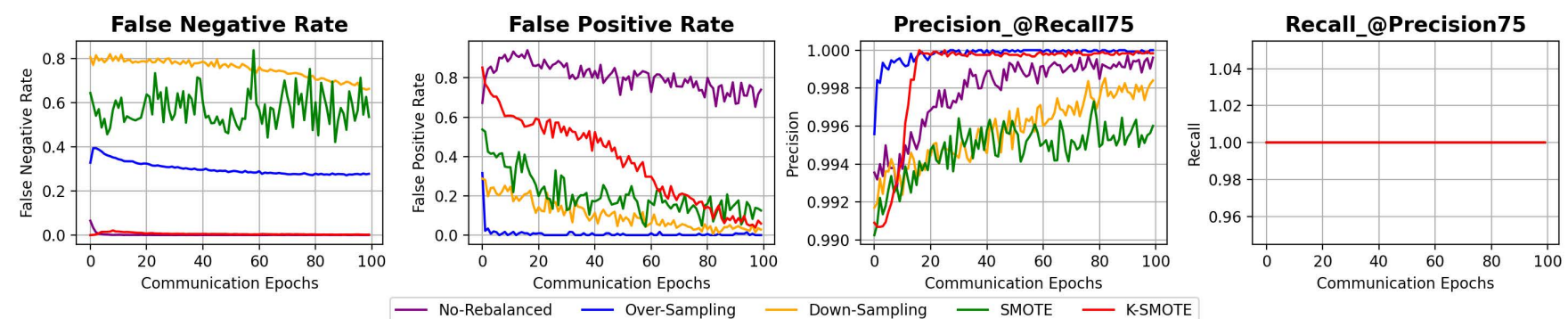

Figure 6: P2PK-SMOTE versus other state-of-the-art methods on different metrics in Experiment 5.2.2 on UNSW-IoT dataset
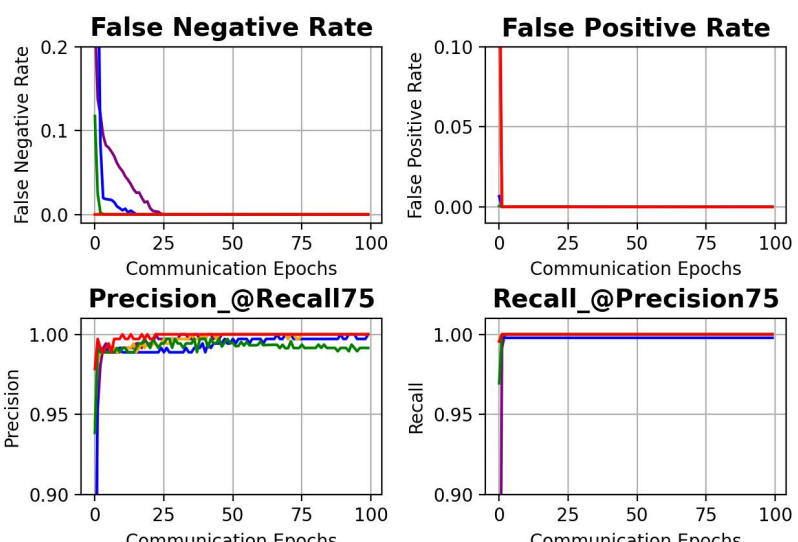

Recall_@Precision75

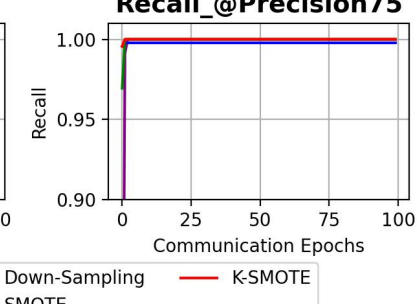

- No-Rebalanced — Down-Sampling - K-SMOTE

— Over-Sampling — SMOTE

Figure 7: P2PK-SMOTE versus other state-of-the-art methods on different metrics in Experiment 5.2.3

in the end of training. Although Over-sampling performs slightly better in precision and has low value in FPR, its FNR converges at near $0.3 \%$. Note that because the dataset contains much more positive, recall does not show much information with every methods. this set of experiments reflects that P2PK-SMOTE is more adaptive to real-world situation, and more efficient to re-balance every local dataset on different clients via the P2P framework.
Table 3: Traffic distribution on clients for Experiment 5.2.4

\begin{tabular}{|c|c|c|c|}
\hline \multicolumn{4}{|c|}{ N-BaIoT } \\
\hline & Client A & Client B & Client C \\
\hline & $\begin{array}{c}\text { PT } 838 \\
\text { Security Camera }\end{array}$ & $\begin{array}{l}\text { XCS7 } 1002 \text { WHT } \\
\text { Security Camera }\end{array}$ & $\begin{array}{l}\text { Danmini } \\
\text { Doorbell }\end{array}$ \\
\hline Train_benign & 11821 & 5590 & 7927 \\
\hline Train_anomaly & 23 & 66 & 102 \\
\hline Global_benign & \multicolumn{3}{|c|}{5067} \\
\hline Global_anomaly & \multicolumn{3}{|c|}{37} \\
\hline \multicolumn{4}{|c|}{ UNSW BoT-IoT } \\
\hline & Client A & Client B & Client C \\
\hline & Scan attack & DoS attack & DDoS attack \\
\hline Train_benign & 38 & 47 & 38 \\
\hline Train_anomaly & 3187 & 5775 & 6743 \\
\hline Global_benign & \multicolumn{3}{|c|}{52} \\
\hline Global_anomaly & \multicolumn{3}{|c|}{10099} \\
\hline
\end{tabular}

\section{SECURITY ANALYSIS}

P2PK-SMOTE is designed for data re-balancing in P2PFL framework, especially addressing the issue of non-IID data distribution in IoT network. The cause of this non-IID phenomenon is heterogeneity of various IoT devices, and this phenomenon degrades the model's performance in FL. It is bound to sacrifice some privacy to solve the problem of non-IID. However, P2PK-SMOTE creates a winwin way, which can solve the problem, but also without losing privacy during the collaborative learning task. We propose to share some artificial data between some participants to help their local data re-balancing. K-SMOTE in P2PK-SMOTE brings out a better 

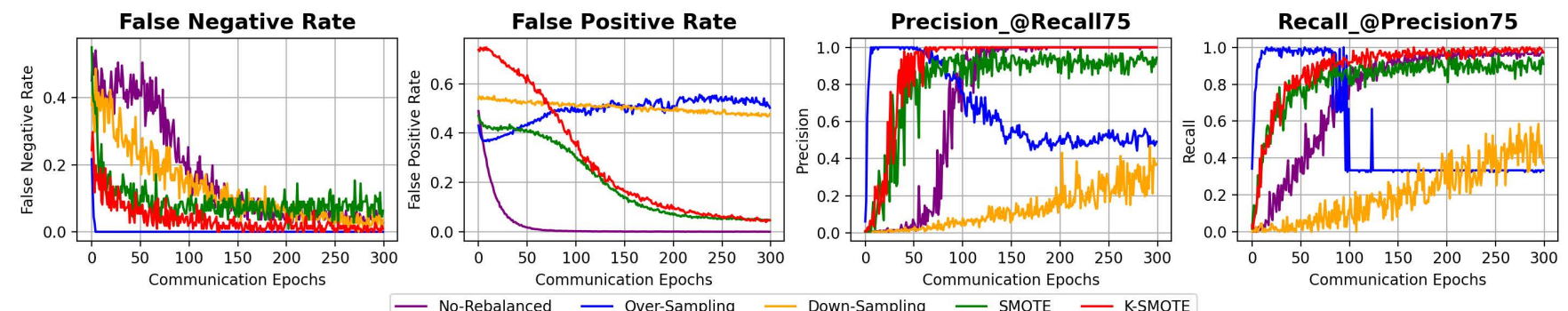

Figure 8: Comparison of different metrics in Experiment 5.2.4 on N-BaIoT
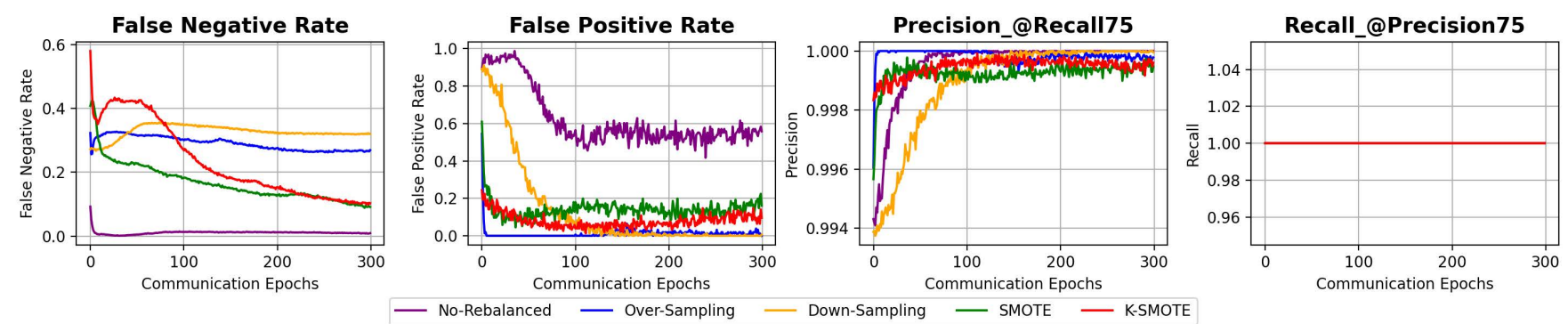

Figure 9: Comparison of different metrics in Experiment 5.2.4 on UNSW Bot-IoT

way to generate artificially synthetic points that can be shared, but more difficult to be identified by the third parties. Moreover, with the help by P2PFL, the synthetic points are not shared with all participants but only some specific devices, since there is no central node/cloud being a coordinator to maintain or control the FL process. This actually brings more privacy to each participated clients, as there is no globally shared model that can be accessed by any device in the FL setup. Sharing these synthetic data points is beneficial especially in non-IID scenarios, as the data from the minority class can be very scarce and from different nature, e.g. different devices can suffer different types of attacks. Thus, when using standard approaches with no sharing of information, this can lead to problems in the model's convergence and performance, as the model may not be able to capture certain types of attacks or may have limited capacity to predict small variations of existing attacks. However, when sharing some information about the anomalies, we enable the creation of a broader variety of synthetic points that enrich the local training datasets, mitigating (at least partially) the aforementioned problems.

In the assumption that there are no compromised clients in FL set up, P2PK-SMOTE brings a new anomaly detection method that is adaptive to imbalanced and non-IID data in IoT network. From security perspective, P2PK-SMOTE is more reliable and trustworthy. However, it consider any adversarial attack targeting the learning algorithm itself, both at training or at test time. For this, at training time, robust aggregation schemes, similar to those used in FL [3, 23, 30], are needed to defend against data and model poisoning attacks [1]. At test time, we can resort to adversarial training [20] to mitigate the impact of adversarial examples aiming to evade detection $[2,28]$. The combination of these techniques to defend against such attacks with the re-balancing mechanisms proposed in this paper is left for future work.

\section{CONCLUSION AND FUTURE WORK}

In this paper, we have introduced P2PK-SMOTE, a data re-balancing approach that is adaptively applied in peer-to-peer federated learning for anomaly detection. P2PK-SMOTE is especially designed for addressing imbalanced and non-IID data in IoT network, thus it proposes to share complex synthetic points that are artificial generated by different clients participating in FL. We have improved SMOTE by having more than one nearest neighbors to compute linear interpolation for generating synthetic points, which makes the points more unpredictable and ready to share. We have further evaluated P2PK-SMOTE on two real-world datasets using three state-of-theart re-balancing methods. We have investigated the performance of P2PK-SMOTE in 4 scenarios, and P2PK-SMOTE proofs its effectiveness by empirical results. It achieves $100 \%$ in recall and precision, and almost $0 \%$ in false negative and false positive.

Despite the benefits P2PK-SMOTE brings, P2PK-SMOTE is still vulnerable to the adversarial attack launched by any participants in FL. Therefore, it is necessary to have a robust method to strengthen the trust among connected clients, and how to detect compromised clients. For the future work, we plan to develop a method that is able to weight the clients for a robust aggregation in order to defend against adversarial attack. We also plan to study what kind of network topology can help saving more privacy but upgrading model performance in peer-to-peer federated learning. 


\section{ACKNOWLEDGEMENTS}

This research has been partly supported by the EC H2020 Project CONCORDIA GA 830927; partly by the RISE Cybersecurity KP;and partly by the EC H2020 nIoVe GA 833742.

\section{REFERENCES}

[1] Arjun Nitin Bhagoji, Supriyo Chakraborty, Prateek Mittal, and Seraphin Calo. 2019. Analyzing Federated Learning through an Adversarial Lens. In ICML. 634-643.

[2] Battista Biggio, Igino Corona, Davide Maiorca, Blaine Nelson, Nedim Šrndić, Pavel Laskov, Giorgio Giacinto, and Fabio Roli. 2013. Evasion Attacks against Machine Learning at Test Time. In foint European Conference on Machine Learning and Knowledge Discovery in Databases. 387-402.

[3] Peva Blanchard, Rachid Guerraoui, Julien Stainer, et al. 2017. Machine Learning with Adversaries: Byzantine Tolerant Gradient Descent. In NeurIPS. 119-129.

[4] Keith Bonawitz, Hubert Eichner, Wolfgang Grieskamp, Dzmitry Huba, Alex Ingerman, Vladimir Ivanov, Chloe Kiddon, Jakub Konečný, Stefano Mazzocchi, H. Brendan McMahan, Timon Van Overveldt, David Petrou, Daniel Ramage, and Jason Roselander. 2019. Towards Federated Learning at Scale: System Design (2019). arXiv:1902.01046 [cs.LG]

[5] Keith Bonawitz, Vladimir Ivanov, Ben Kreuter, Antonio Marcedone, H. Brendan McMahan, Sarvar Patel, Daniel Ramage, Aaron Segal, and Karn Seth. 2017. Practical Secure Aggregation for Privacy-Preserving Machine Learning. (2017), 1175-1191. https://doi.org/10.1145/3133956.3133982

[6] Nitesh V. Chawla. 2005. Data Mining for Imbalanced Datasets: An Overview. Springer US, Boston, MA, 853-867. https://doi.org/10.1007/0-387-25465-X_40

[7] Nitesh V. Chawla, Kevin W. Bowyer, Lawrence O. Hall, and W. Philip Kegelmeyer 2002. SMOTE: Synthetic Minority over-Sampling Technique. F. Artif. Int. Res. 16 , 1 (June 2002), 321-357.

[8] Jorge de la Calleja and Olac Fuentes. 2007. A Distance-Based Over-Sampling Method for Learning from Imbalanced Data Sets. (2007).

[9] Moming Duan. 2019. Astraea: Self-Balancing Federated Learning for Improving Classification Accuracy of Mobile Deep Learning Applications. 2019 IEEE 37th International Conference on Computer Design (ICCD) (2019), 246-254.

[10] Alberto Fernández, Salvador García, Francisco Herrera, and Nitesh V. Chawla. 2018. SMOTE for Learning from Imbalanced Data: Progress and Challenges, Marking the 15-Year Anniversary. F. Artif. Int. Res. 61, 1 (Jan. 2018), 863-905.

[11] Arya Iranmehr, Hamed Masnadi-Shirazi, and Nuno Vasconcelos. 2019. Costsensitive support vector machines. Neurocomputing 343 (May 2019), 50-64. https://doi.org/10.1016/j.neucom.2018.11.099

[12] Eunjeong Jeong, Seungeun Oh, Hyesung Kim, Jihong Park, Mehdi Bennis, and Seong-Lyun Kim. 2018. Communication-Efficient On-Device Machine Learning Federated Distillation and Augmentation under Non-IID Private Data. ArXiv abs/1811.11479 (2018)

[13] Jakub Konecný, Brendan McMahan, and Daniel Ramage. 2015. Federated Optimization: Distributed Optimization Beyond the Datacenter. CoRR abs/1511.03575 (2015). arXiv:1511.03575 http://arxiv.org/abs/1511.03575

[14] Jakub Konečný, H. Brendan McMahan, Felix X. Yu, Peter Richtárik, Ananda Theertha Suresh, and Dave Bacon. 2016. Federated Learning: Strategies for Improving Communication Efficiency. arXiv:1610.05492 [cs.LG]

[15] Kavya Kopparapu and Eric Lin. 2020. FedFMC: Sequential Efficient Federated Learning on Non-iid Data. ArXiv abs/2006.10937 (2020).

[16] Nickolaos Koroniotis, Nour Moustafa, Elena Sitnikova, and Benjamin Turnbull. 2019. Towards the development of realistic botnet dataset in the Internet of Things for network forensic analytics: Bot-IoT dataset. Future Generation Computer Systems 100 (2019), 779-796. https://doi.org/10.1016/j.future.2019.05.041

[17] Tian Li, Anit Kumar Sahu, Ameet Talwalkar, and Virginia Smith. 2019. Federated Learning: Challenges, Methods, and Future Directions. (2019). arXiv:1908.07873 [cs.LG]

[18] Tian Li, Anit Kumar Sahu, Manzil Zaheer, Maziar Sanjabi, Ameet Talwalkar, and Virginia Smith. 2018. Federated Optimization in Heterogeneous Networks. arXiv:1812.06127 [cs.LG]

[19] Lumin Liu, Jun Zhang, S. H. Song, and Khaled Ben Letaief. 2019. Edge-Assisted Hierarchical Federated Learning with Non-IID Data. CoRR abs/1905.06641 (2019). arXiv:1905.06641 http://arxiv.org/abs/1905.06641

[20] Aleksander Madry, Aleksandar Makelov, Ludwig Schmidt, Dimitris Tsipras, and Adrian Vladu. 2018. Towards Deep Learning Models Resistant to Adversarial Attacks. In International Conference on Learning Representations (ICLR).

[21] H. Brendan McMahan, Eider Moore, Daniel Ramage, Seth Hampson, and Blaise Agüera y Arcas. 2016. Communication-Efficient Learning of Deep Networks from Decentralized Data. arXiv:1602.05629 [cs.LG]

[22] Y. Meidan, M. Bohadana, Y. Mathov, Y. Mirsky, A. Shabtai, D. Breitenbacher, and Y. Elovici. 2018. N-BaIoT-Network-Based Detection of IoT Botnet Attacks Using Deep Autoencoders. IEEE Pervasive Computing 17, 3 (2018), 12-22.
[23] Luis Muñoz-González, Kenneth T Co, and Emil C Lupu. 2019. Byzantine-Robust Federated Machine Learning through Adaptive Model Averaging. arXiv preprint arXiv:1909.05125 (2019).

[24] Thien Duc Nguyen, Samuel Marchal, Markus Miettinen, Hossein Fereidooni, N. Asokan, and Ahmad-Reza Sadeghi. 2019. DÏoT: A Federated Self-learning Anomaly Detection System for IoT. 2019 IEEE 39th International Conference on Distributed Computing Systems (ICDCS) (Jul 2019). https://doi.org/10.1109/icdcs. 2019.00080

[25] Abhijit Guha Roy, Shayan Siddiqui, Sebastian Pölsterl, Nassir Navab, and Christian Wachinger. 2019. BrainTorrent: A Peer-to-Peer Environment for Decentralized Federated Learning. CoRR abs/1905.06731 (2019). arXiv:1905.06731 http://arxiv.org/abs/1905.06731

[26] Felix Sattler, Simon Wiedemann, Klaus-Robert Müller, and Wojciech Samek. 2019. Robust and Communication-Efficient Federated Learning from Non-IID Data. CoRR abs/1903.02891 (2019). arXiv:1903.02891 http://arxiv.org/abs/1903.02891

[27] Amira Soliman, Sarunas Girdzijauskas, M.-R Bouguelia, Sepideh Pashami, and Slawomir Nowaczyk. 2020. Decentralized and Adaptive K-Means Clustering for Non-IID Data Using HyperLogLog Counters. Advances in Knowledge Discovery and Data Mining: 24th Pacific-Asia Conference, PAKDD (05 2020), 343-355. https: //doi.org/10.1007/978-3-030-47426-3_27

[28] Christian Szegedy, Wojciech Zaremba, Ilya Sutskever, Joan Bruna, Dumitru Erhan, Ian Goodfellow, and Rob Fergus. 2013. Intriguing Properties of Neural Networks. arXiv preprint arXiv:1312.6199 (2013).

[29] Shiqiang Wang, Tiffany Tuor, Theodoros Salonidis, Kin K. Leung, Christian Makaya, Ting He, and Kevin Chan. 2018. When Edge Meets Learning: Adaptive Control for Resource-Constrained Distributed Machine Learning. CoRR abs/1804.05271 (2018). arXiv:1804.05271 http://arxiv.org/abs/1804.05271

[30] Dong Yin, Yudong Chen, Kannan Ramchandran, and Peter Bartlett. 2018. Byzantine-Robust Distributed Learning: Towards Optimal Statistical Rates. In ICML. 5636-5645.

[31] Naoya Yoshida, Takayuki Nishio, Masahiro Morikura, Koji Yamamoto, and Ryo Yonetani. 2019. Hybrid-FL for Wireless Networks: Cooperative Learning Mechanism Using Non-IID Data. (2019). arXiv:1905.07210 [cs.LG]

[32] J. Zhang and I. Mani. 2003. KNN Approach to Unbalanced Data Distributions: A Case Study Involving Information Extraction. (2003).

[33] Yue Zhao, Meng Li, Liangzhen Lai, Naveen Suda, Damon Civin, and Vikas Chandra. 2018. Federated Learning with Non-IID Data. (2018). arXiv:1806.00582 [cs.LG] 